\title{
Estudo Longitudinal EpiFloripa Idoso - Rotinas de organização e protocolos referentes à coleta, análise e armazenamento de material biológico, exames de imagem e capacidade físico-funcional
}

\section{EpiFloripa Ageing Longitudinal Study - Organizational} routines and protocols related to the collection, analysis and storage of biological material, image exams and physical-functional capacity

Susana Cararo Confortin ${ }^{1 *}$ (1), Ione Jayce Ceola Schneider ${ }^{1,2}$ (D), Ana Lúcia Danielewicz ${ }^{1}$ (1), Angélica Scherlowski Fassula ${ }^{3}$ (D), Bianca Bittencourt de Souza ${ }^{1}$ (1), Carla Elane Silva dos Santos ${ }^{4}$ (1), Danielle Ledur Antes ${ }^{5}$ (D), Francieli Cembranel ${ }^{1}$ (1), Karine Gonçalves Pereira' ${ }^{1}$ (1), Lariane Mortean Ono (i), Larissa Pruner Marques ${ }^{1}$ (1), Lucélia Justino Borges ${ }^{7}$ (1), Rodrigo de Rosso $\mathrm{Krug}^{8}$ (1), Isabela de Carlos Back $^{9}$ (1), Tania Rosane Bertoldo Benedetti10 (10), Cassiano Ricardo Rech ${ }^{10}$ (1), Aline Rodrigues Barbosa ${ }^{10}$ (i), Eleonora d'Orsi' ${ }^{1}$ (i)

\footnotetext{
${ }_{1}^{1}$ Programa de Pós-graduação em Saúde Coletiva, Universidade Federal de Santa Catarina (UFSC) - Florianópolis (SC), Brasil.

${ }^{2}$ Curso de Fisioterapia, Universidade Federal de Santa Catarina (UFSC) - Araranguá (SC), Brasil.

${ }^{3}$ Programa de Pós-graduação em Nutrição, Universidade Federal de Santa Catarina (UFSC) - Florianópolis (SC), Brasil.

${ }^{4}$ Curso de Especialização em Saúde Coletiva, Universidade Federal de Santa Catarina (UFSC) - Florianópolis (SC), Brasil.

${ }^{5}$ Complexo de Ensino Superior Meridional (IMED) - Passo Fundo (RS), Brasil.

${ }^{6}$ Programa de Pós-graduação em Ciências Médicas, Universidade Federal de Santa Catarina (UFSC) - Florianópolis (SC), Brasil.

${ }^{7}$ Departamento de Educação Física, Universidade Federal do Paraná (UFPR) - Curitiba (PR), Brasil.

${ }^{8}$ Centro de Ciências da Saúde e Agrárias, Universidade de Cruz Alta (UNICRUZ) - Cruz Alta (RS), Brasil

${ }^{9}$ Departamento de Pediatria, Universidade Federal de Santa Catarina (UFSC) - Florianópolis (SC), Brasil

${ }^{10}$ Departamento de Educação Física, Universidade Federal de Santa Catarina (UFSC) - Florianópolis (SC), Brasil.

Trabalho realizado com dados de idosos residentes do município de Florianópolis (SC), Brasil.

Endereço para correspondência: Susana Cararo Confortin - Programa de Pós-graduação em Saúde Coletiva, Centro de Ciências da Saúde, Universidade Federal de Santa Catarina (UFSC), Rua Delfino Conti, s/n, Bloco H - Campus Reitor João David Ferreira Lima - CEP: 88040-900 - Florianópolis (SC), Brasil - E-mail: susanaconfortin@gmail.com

Fonte de financiamento: Conselho Nacional de Desenvolvimento Científico e Tecnológico (CNPq), processo nº 475.904/2013-3 e infra-estrutura da UFSC. Conflito de interesses: nada a declarar.
} 


\begin{abstract}
Resumo
Introdução: Exames complementares podem contribuir para a compreensão da etiologia de agravos à saúde de idosos. Objetivo: Descrever os aspectos operacionais e protocolos referentes à coleta, análise e armazenamento de material biológico, exames de imagem e capacidade físico-funcional. Método: Estudo longitudinal, de base populacional, com 604 idosos ( $\geq 60$ anos) do estudo EpiFloripa Idoso, em 2014. Foram coletados dados de material biológico, composição corporal, densitometria óssea, ultrassonografia da espessura íntima média das artérias carótidas, força de preensão manual e a atividade física. Resultados: A taxa de resposta foi de $50,4 \%$. Houve perda de seguimento em relação à faixa etária, estado cognitivo e atividades de vida diária. Os indivíduos que realizaram os exames eram mais novos, trabalhavam no momento da entrevista, ingeriam álcool, eram fisicamente ativos. Além de apresentarem menor grau de dependência, ausência de déficit cognitivo, ausência de sintomas depressivos e ausência de dificuldade na mobilidade. Conclusão: Espera-se que a descrição das rotinas aplicadas possa auxiliar no desenvolvimento de novas pesquisas semelhantes em grupos de idosos. Assim, poderá ser acompanhado o processo de envelhecimento da população, seus fatores de risco e proteção.
\end{abstract}

Palavras-chaves: estudos longitudinais; métodos; idoso.

\begin{abstract}
Background: Complementary exams may contribute to the understanding of the etiology of health problems in older adults. Objective: To describe the operational aspects and protocols used to collect, analyze and storage biological materials, image exams and physical-functional capacity tests. Method: This is a longitudinal and population-based study with 604 older adults ( $\geq$ 60 years) from EpiFloripa Ageing Study, in 2014. Data of biological material, body composition, bone densitometry, ultrasonography of the carotid artery intima average thickness, hand grip strength and physical activity were collected. Results: The response rate was $50.4 \%$. There was loss of follow-up in relation to age, cognitive status, and activities of daily living. The individuals who performed the tests were younger, worked by the time of the interview, ingested alcohol and were physically active. Also, they were less dependent, absence of cognitive impairment, absence of depressive symptoms and absence of difficulty in mobility. Conclusion: It is expected that the description of the applied routines may help in the development of new similar research in the older adult population. Thus, the aging process of the population, its risk and protection factors can be followed.
\end{abstract}

Keywords: longitudinal studies; methods; older adults.

\section{INTRODUÇÃO}

Estudos epidemiológicos sobre o envelhecimento da população tornam-se importantes, especialmente nos países de média e baixa renda, nos quais tal processo vem ocorrendo de forma acelerada ${ }^{1}$. A partir desses estudos, pode-se estimar a prevalência de doenças, identificar fatores e comportamentos protetores ou de risco e orientar estratégias preventivas ${ }^{2}$, como a prática de atividade física, alimentação saudável, realização de consultas e exames regulares, dentre outras, adotadas com base nos resultados de grandes estudos ${ }^{3-5}$. Além disso, a realização de exames laboratoriais, de imagem e de capacidade físico-funcional exerce papel importante na avaliação de saúde dos idosos, devido à possibilidade de avaliação direta das alterações fisiopatológicas observadas no processo de envelhecimento.

Em estudos epidemiológicos, medidas e informações autorreportadas podem apresentar viés de informação ${ }^{6}$. A realização de exames complementares pode contribuir para a compreensão da etiologia de agravos à saúde de idosos. Contudo, a realização dessas medidas necessita de maior financiamento, infraestrutura ${ }^{7}$, planejamento, equipe multidisciplinar, o que pode tornar a condução complexa e de difícil execução. Na América Latina, ainda são poucos os estudos longitudinais de base populacional sobre envelhecimento ${ }^{8-10}$, e as investigações clínicas, a partir das linhas de base dessas coortes, ainda são incipientes ${ }^{7}$.

Evidências epidemiológicas sobre a saúde da população idosa são fundamentais para elaborar políticas públicas e programas que atendam às especificidades deste grupo populacional. Assim, ao considerar o estudo longitudinal EpiFloripa Idoso, que avaliou as condições de saúde dos idosos de Florianópolis/Santa Catarina, o presente manuscrito teve por objetivo descrever os aspectos operacionais, as estratégias e os protocolos de coleta, análise e armazenamento de material biológico, de exames de imagem e de capacidade físico-funcional realizados na segunda onda deste estudo.

\section{MÉTODO}

O EpiFloripa Idoso é um estudo longitudinal, de base populacional, realizado em 2013/2014, como seguimento ao estudo "Condições de saúde da população idosa do município de Florianópolis/SC: estudo de base populacional - EpiFloripa Idoso", que teve sua linha de base em 2009/2010.

O estudo foi realizado na zona urbana do município de Florianópolis, capital do estado de Santa Catarina, região Sul do Brasil. O município possui elevado índice de desenvolvimento humano municipal (IDH-M $=0,847$ ), considerado o terceiro melhor IDH-M do país ${ }^{11}$. Além disso, apresenta moderada esperança de vida ao nascer $(77,4 \text { anos })^{12}$. Em 2010, o Índice GINI de renda familiar do município era de $0,547^{13}$.

Os métodos para o planejamento amostral da linha de base (2009/2010) e seguimento (2013/2014) foram previamente publicados ${ }^{14}$. Os idosos que participaram do seguimento, entre 2013 e 2014, foram convidados para a etapa seguinte do estudo, que era a realização de exames complementares nas dependências do Centro de Ciências da Saúde da Universidade Federal de 
Santa Catarina (UFSC), a fim de suplementar as investigações do EpiFloripa Idoso 2013/2014.

Os objetivos da pesquisa foram ampliados no seguimento e realizados coleta, análise e armazenamento de material biológico (sangue), avaliação da composição corporal (DXA), mensuração do nível de atividade física objetiva (acelerômetro), da força de preensão manual (FPM) e ultrassom da carótida. Para tanto, a partir de março de 2014, todos os idosos que responderam à entrevista domiciliar do seguimento $(\mathrm{n}=1.197)$ foram contatados por telefone e convidados a participar desta etapa. A etapa dos exames envolveu 604 idosos.

O processo de agendamento dos exames foi realizado via contato telefônico por duas pesquisadoras do estudo. A ordem das ligações obedeceu à sequência de realização das entrevistas e ao interesse demonstrado pelos idosos em participarem desta etapa. Entre os meses de março e julho (2014), foram agendados, em média, 12 idosos/dia, em três dias da semana. A partir de agosto e até dezembro de 2014, o número médio de entrevistas diárias se elevou para 15, e a coleta foi restrita a um dia na semana. Durante o agendamento, todos idosos foram orientados em relação ao local de realização e horário de início dos exames (7h30min da manhã), necessidade de jejum (mínimo de 8 e máximo de 10 horas) e apresentação de documento oficial de identificação com foto.

Nos casos de recusa durante o primeiro contato telefônico, foram realizadas duas novas tentativas em momentos distintos, e somente após o terceiro contato sem sucesso os participantes que se negaram a realizar os exames foram considerados recusas. O mesmo procedimento foi adotado para os participantes agendados e que faltaram aos exames. Assim, aqueles que não compareceram após o terceiro agendamento consecutivo foram considerados recusas.

Em relação à logística de trabalho de campo, ao chegarem ao local do estudo, os participantes foram recepcionados por um dos membros da equipe, que os acompanhavam até o local da realização dos exames. Os objetivos da pesquisa foram explicitados aos participantes, como parte do processo de consentimento de participação, e o participante ou seu responsável legal assinava o Termo de Consentimento Livre e Esclarecido (TCLE) e assinalava os exames que consentiu participar.

Posteriormente, os idosos receberam um crachá de controle, o qual continha etiquetas com os nomes de todos os exames a serem realizados. Após o término de cada exame, o avaliador retirava a etiqueta correspondente.

O primeiro exame era normalmente a coleta de material biológico, principalmente devido ao jejum, que ocorria no laboratório do $4^{\circ}$ andar do Departamento de Nutrição do Centro de Ciências da Saúde da UFSC. Em seguida era oferecido café da manhã. Na sequência, os idosos eram encaminhados ao laboratório de antropometria do prédio do Centro Ciências da Saúde da UFSC, onde realizavam as demais avaliações (ultrassom da artéria carótida, DXA, FPM) e para a orientação quanto ao uso do acelerômetro. Cada procedimento era realizado em uma sala particular, e o encaminhamento dos participantes seguia um sistema de revezamento que visava minimizar o tempo de espera, sempre respeitando a ordem de chegada dos participantes.

Após a realização dos procedimentos e orientação em relação ao uso dos acelerômetros, os participantes recebiam um brinde do estudo e eram dispensados.

A equipe de trabalho foi previamente capacitada para a realização dos procedimentos da pesquisa e era composta por discentes de graduação e pós-graduação (mestrado, doutorado e pós-doutorado), com formação em diferentes áreas da saúde (educação física, enfermagem, farmácia, fisioterapia e nutrição), dois professores do Departamento de Saúde Pública e Ciências Médicas - UFSC e pela professora coordenadora do estudo.

O período de realização das coletas foi de março a dezembro de 2014. Todas as coletas aconteceram no período da manhã, nas dependências da UFSC. Entre os meses de março e julho, os entrevistadores se organizaram em três equipes de trabalho, que se alternavam diariamente para realizar as avaliações. Cada uma das equipes era composta por sete discentes e um médico responsável pelo exame de ultrassom da artéria carótida. A professora coordenadora do estudo supervisionava as coletas de todas as equipes.

Cada membro da equipe era responsável por funções específicas dentre as estabelecidas previamente: recepção dos idosos, processo de consentimento em participar do estudo, organização do café da manhã, coleta de material biológico, DXA, ultrassonografia de carótida, orientação e entrega de acelerômetro e teste de FPM.

\section{Coleta, análise e armazenamento de material biológico}

O procedimento de coleta de material biológico constou da retirada de amostras sanguíneas. Foi realizado no laboratório da UFSC, entre $7 \mathrm{~h} 30 \mathrm{~min}$ e $9 \mathrm{~h} 30 \mathrm{~min}$, e seguiu o protocolo do Laboratório do Hospital Universitário da UFSC. A pessoa responsável por auxiliar nesta etapa fazia a identificação do participante, etiquetagem dos tubos e orientação sobre o recebimento dos resultados, o que evitou erros de identificação e garantiu a qualidade da coleta.

Foram coletados $30 \mathrm{~mL}$ de sangue venoso periférico de cada participante, após jejum de 8 a 10 horas. Uma parte das amostras sanguíneas foi analisada pelo Laboratório do Hospital Universitário da UFSC, outra foi armazenada em tubos criogênicos (alíquotas de soro e plasma) para posteriores análises, em ultrafreezer a $-80{ }^{\circ} \mathrm{C}$. Para controle e posterior 
localização dos tubos, foi elaborado um mapa das caixas no programa Microsoft Office Excel.

Os exames bioquímicos realizados neste estudo, o método, kit utilizado e os parâmetros foram descritos a seguir no Quadro 1.

Os dados referentes aos resultados dos exames de cada participante foram duplamente digitados em arquivo Excel, a cada semana, para posteriores controles de qualidade e análises estatísticas. No caso de divergência, uma terceira pessoa realizou o acesso aos exames de sangue para conferência do valor.

\section{Composição corporal e densitometria óssea}

As avaliações da composição corporal e densitometria óssea foram realizadas por meio do densitômetro de dupla emissão com fonte de raios X (DXA - Dual Energy X-ray Absorptiometry, Modelo Lunar Prodigy Advance da General Electric). Para verificar/garantir a funcionalidade e a precisão do densitômetro, realizou-se o procedimento diário e semanal de garantia de qualidade, com objeto-padrão próprio para massa óssea e outro para as partes moles, de acordo com as recomendações do aparelho/fabricante ${ }^{21}$.

Quadro 1. Descrição do método, kit e parâmetros utilizados para os exames bioquímicos. Florianópolis, Santa Catarina, Brasil, 2014/2015

\begin{tabular}{|c|c|c|}
\hline Exame & Método e kit & Parâmetros \\
\hline Colesterol Total & $\begin{array}{l}\text { Enzimático colorimétrico bicromático de ponto final } \\
\text { automatizado, kit Flex }{ }^{\circledR} \text { Reagent Cartridge CHOL }\end{array}$ & $\begin{array}{l}\text { Desejável < } 200 \mathrm{mg} / \mathrm{dL} \\
\text { Limítrofe } 200 \text { a } 239 \mathrm{mg} / \mathrm{dL} \\
\text { Alto } \geq 240 \mathrm{mg} / \mathrm{dL}^{15}\end{array}$ \\
\hline Triglicerídeos & $\begin{array}{l}\text { Enzimático colorimétrico bicromático de ponto final } \\
\text { automatizado, kit } \\
\text { Flex }^{\circledR} \text { Reagent Cartridge TC }\end{array}$ & $\begin{array}{l}\text { Ótimo }<150 \mathrm{mg} / \mathrm{dL} \\
\text { Limítrofe } 150 \text { a } 200 \mathrm{mg} / \mathrm{dL} \\
\text { Alto } 201 \text { a } 499 \mathrm{mg} / \mathrm{dL} \text { e } \\
\text { Muito alto } \geq 500 \mathrm{mg} / \mathrm{dL}^{15}\end{array}$ \\
\hline HDL-Colesterol & $\begin{array}{l}\text { Detergente seletivo acelerador, kit Flex }{ }^{\circledR} \text { Reagent Cartridge } \\
\text { AHDL }\end{array}$ & $\begin{array}{c}\text { Baixa }<40 \mathrm{mg} / \mathrm{dL} \\
\text { Limítrofe } 40 \text { a } 60 \mathrm{mg} / \mathrm{dL} \text { e } \\
\text { Desejável }>60 \mathrm{mg} / \mathrm{dL}^{15}\end{array}$ \\
\hline LDL-Colesterol & $\begin{array}{l}\text { Automatizado de precipitação de lipoproteínas de baixa } \\
\text { densidade, kit Flex }{ }^{\circledR} \text { Reagent Cartridge ALDL }\end{array}$ & $\begin{array}{c}\text { Ótima < } 100 \text { mg/dL; } \\
\text { Desejável } 100 \text { a } 129 \text { mg/dL; } \\
\text { Limítrofe } 130 \text { a } 159 \mathrm{mg} / \mathrm{dL} ; \\
\text { Alta } 160 \text { a } 189 \text { mg/dL e } \\
\text { Muito alta } \geq 190 \mathrm{mg} / \mathrm{dL}^{15}\end{array}$ \\
\hline $\begin{array}{l}\text { Índices I e II de Castelli } \\
\text { (CT/HDL e LDL/HDL) }\end{array}$ & & $\begin{array}{l}\text { I - Homens desejável até } 4,9 \text {; } \\
\text { Mulheres até } 4,3^{16} \text {. } \\
\text { II - Homens desejável até } 3,3 \text {; } \\
\text { Mulheres até } 2,9^{16} \text {. }\end{array}$ \\
\hline Glicose & $\begin{array}{l}\text { Adaptação do método hexoquinase-glicose-6-fosfato } \\
\text { desidrogenase, kit cartucho Flex }{ }^{\circledR} \text { Reagent Cartridge GLUC }\end{array}$ & $\begin{array}{l}\text { Normal até } 99 \mathrm{mg} / \mathrm{dL} \\
\text { Alterado } 100 \text { a } 125 \mathrm{mg} / \mathrm{dL} \text { e } \\
\text { Alto } \geq 126 \mathrm{mg} / \mathrm{dL}^{17}\end{array}$ \\
\hline Hemoglobina glicada & $\begin{array}{l}\text { Amostra de sangue total, por cromatografia de troca iônica } \\
\text { de alta pressão. }\end{array}$ & $\begin{array}{c}\text { Baixa }<4,3 \% \\
\text { Referência } 4,3 \text { a } 6,1 \% \text { e } \\
\text { Alta }>6,1 \%^{18}\end{array}$ \\
\hline Hemograma completo & $\begin{array}{l}\text { Citometria de fluxo com laser semicondutor, } \\
\text { foco hidrodinâmico e método SLS de detecção da } \\
\text { hemoglobina (absorção espectrofotométrica). }\end{array}$ & $\begin{array}{c}\text { Hemácias - } \\
\text { Homens 4,5 a 6,0 milhões/mm3; } \\
\text { Mulheres } 4 \text { a 5,2 milhões/mm3. } \\
\text { Hemoglobina - } \\
\text { Homens } 13 \text { a } 18 \mathrm{~g} / \mathrm{dL} ; \\
\text { Mulheres } 12 \text { a } 16 \mathrm{~g} / \mathrm{dL} . \\
\text { Hematócrito - } \\
\text { Homens } 40,0 \text { a } 52,0 \% ; \\
\text { Mulheres } 37,0 \text { a } 47,0 \% . \\
\text { Leucócitos } 3800 \text { e } 11000 \mathrm{~mm} 3 . \\
\text { Plaquetas } 150000 \text { a } 400000 \mathrm{~mm} 3^{19} .\end{array}$ \\
\hline Ácido Fólico & $\begin{array}{l}\text { Imunoensaio competitivo por quimioluminescência, kit } \\
\text { Folic Acid Immulite }^{\circledR} 2000\end{array}$ & $\begin{array}{l}\text { Baixo }<3 \mathrm{ng} / \mathrm{mL} \\
\text { Normal } 3 \text { a } 17 \mathrm{ng} / \mathrm{mL} \text { e } \\
\text { Alto }>17 \mathrm{ng} / \mathrm{mL} \\
\end{array}$ \\
\hline $\begin{array}{l}\text { Proteína C-Reativa de alta } \\
\text { sensibilidade }\end{array}$ & Imunonefelometria & $\begin{array}{l}\text { Baixo risco }<1,0 \mathrm{mg} / \mathrm{L} \\
\text { Médio risco } 1,0 \text { a } 3,0 \mathrm{mg} / \mathrm{L} \text { e } \\
\text { Alto risco }>3,0 \mathrm{mg} / \mathrm{L}^{20}\end{array}$ \\
\hline Vitamina B-12 & $\begin{array}{l}\text { Reação de ensaio competitivo enzimático de fase sólida } \\
\text { por quimioluminescência, kit Vitamin B12 Immulite }{ }^{\circledR} 2000\end{array}$ & $\begin{array}{c}\text { Baixo }<193 \mathrm{pg} / \mathrm{mL} \\
\text { Normal } 193 \text { a } 982 \mathrm{pg} / \mathrm{mL} \text { e } \\
\text { Alto }>982 \mathrm{pg} / \mathrm{mL}\end{array}$ \\
\hline
\end{tabular}


Os dados coletados no DXA incluíram informações para determinação da gordura corporal relativa (\% gordura), massa de gordura (MG em kg), massa magra (MM em kg), conteúdo mineral ósseo (CMO), densidade mineral óssea (DMO), densidade mineral óssea da coluna lombar (DMOCL) e densidade mineral óssea do colo do fêmur (DMOCF). Para a DMO, adotou-se a classificação apresentada no Quadro 2.

A equipe responsável por essa coleta realizou procedimentos de treinamento com um especialista na área para padronização das medidas realizadas.

No que se refere ao procedimento de avaliação, ao entrar na sala de avaliação, era solicitado ao idoso que removesse todos os seus objetos pessoais de metal, os quais eram colocados em embalagem plástica, lacrados, e o idoso era convidado a guardar este em sua bolsa, para não haver perdas. Durante esse procedimento também se explicava o objetivo do exame ao participante.

Enquanto o avaliador posicionava o idoso, explanava as instruções de que deveria permanecer imóvel e não poderia conversar durante a avaliação, as quais eram repetidas caso houvesse dúvidas. $\mathrm{O}$ exame foi realizado procedendo à varredura total do corpo do idoso, densitometria da coluna lombar e densitometria do colo do fêmur. O procedimento total durava, em média, 15 minutos.

Idosos com estatura igual ou superior a 192,5 centímetros e/ou massa corporal igual ou superior a 136,4 kg deveriam ser excluídos do exame, devido às limitações do aparelho. Contudo, não houve nenhum idoso com essas características.

Para realização da varredura total do corpo, o idoso deveria estar centralizado na mesa de exame, em decúbito dorsal, de acordo com a linha central da mesa do DXA, a qual é referência para o alinhamento do corpo. Os braços estendidos sobre a mesa ao longo do corpo, em rotação lateral, com polegares para cima

Quadro 2. Classificação para a densidade mineral óssea de acordo com o sexo

\begin{tabular}{|l|c|c|}
\hline & Mulher & Homem \\
\hline DMO total & & \\
\hline Normal & Acima de 1,045 & Acima de 1,140 \\
\hline Osteopenia & De 1,045 a 0,885 & De 1,140 a 0,980 \\
\hline Osteoporose & Abaixo de 0,885 & Abaixo de 0,980 \\
\hline DMO Coluna lombar & & \\
\hline Normal & Acima de 1,075 & Acima de 1,116 \\
\hline Osteopenia & De 1,075 a 0,895 & De 1,116 a 0,876 \\
\hline Osteoporose & Abaixo de 0,895 & Abaixo de 0,876 \\
\hline DMO Colo Fêmur & & \\
\hline Normal & Acima de 0,882 & Acima de 0,957 \\
\hline Osteopenia & De 0,882 a 0,706 & De 0,957 a 0,669 \\
\hline Osteoporose & Abaixo de 0,706 & Abaixo de 0,669 \\
\hline
\end{tabular}

Legenda: DMO: densidade mineral óssea. A DMO foi calculada a partir da própria amostra deste estudo, sendo considerados, conforme consenso da Organização Mundial da Saúde (OMS) ${ }^{22}$, como normal (até -1 DP da média), osteopenia (de -1 a -2,5 DP) e osteoporose (-2,5 DP para mais) e as palmas das mãos direcionadas para as pernas. A cabeça do idoso era posicionada, aproximadamente, três centímetros abaixo da margem superior da área de varredura da mesa. Os joelhos e os pés do idoso eram contidos com faixas de velcro, a fim de impedir qualquer movimento durante a medição. Caso o idoso fosse maior do que a área de varredura do aparelho, este era posicionado para uma varredura de meio corpo (também conhecida como Mirror Image), conforme instrução do manual do próprio equipamento, o qual estipula ainda que, além de todo o lado direito do corpo, toda a cabeça e a coluna devem estar incluídas na janela de varredura.

Nos casos de dificuldade para manter a posição de decúbito dorsal sem apoio para a cabeça, era oferecido um pequeno travesseiro próprio do aparelho, que não modificava o procedimento realizado.

A posição inicial para a medição da coluna lombar seguiu o mesmo padrão da postura adotada para o corpo inteiro em relação à linha central do DXA, com exceção dos braços, que deveriam estar cruzados sobre o peito e afastados das laterais do quadril, e dos membros inferiores em abdução e rotação interna. Após o posicionamento, utilizava-se uma peça triangular entre os membros inferiores, intitulada correia para pés, para auxiliar na manutenção da postura correta para o exame, não permitindo que o idoso movimentasse as pernas.

Para a avaliação da coluna lombar, posicionava-se o laser cerca de cinco centímetros abaixo da linha do umbigo do idoso e no mesmo plano longitudinal da linha média do corpo. Nos casos em que o idoso apresentava excessiva massa gorda com protuberância abdominal, essa medida de cinco centímetros era descartada, utilizando-se como ponto para o laser o próprio umbigo, levando-se em consideração a visualização da quinta vértebra lombar na imagem. Aqueles idosos que não conseguiam posicionar toda a coluna lombar na mesa de exame, ou com dificuldade para permanecer na posição inicial com os membros inferiores estendidos, foram posicionados com uma almofada em forma de cubo colocada abaixo das pernas, mantendo flexão de quadris e joelhos de aproximadamente 90 graus.

A posição inicial para a medição do fêmur duplo era a mesma posição adotada para a coluna lombar. Para o exame do fêmur duplo (colo do fêmur), a luz do laser do DXA era posicionada, aproximadamente, sete a oito centímetros abaixo do trocânter maior, onde a linha transversal (sínfise púbica) e a linha média do fêmur se intersectam. Avaliava-se primeiro o fêmur esquerdo e, posteriormente, o fêmur direito.

Ao final de cada dia de coleta, o responsável pelos exames realizava a conferência, correção de pontos de referência (se necessária) e salvamento das imagens dos exames no banco de dados da pesquisa, a fim de evitar acúmulo de dados, perda de exames realizados e facilitar o envio dos resultados aos idosos. Os dados numéricos que compunham o banco de dados da pesquisa foram exportados em Microsoft Access e convertidos para Stata, o que evitou erros de digitação. 


\section{Ultrassom de carótida}

O ultrassom de carótida foi realizado utilizando o equipamento Ultrassom Portátil da marca Toshiba, com transdutor setorial. A avaliação foi feita mediante a determinação de três medidas da espessura da íntima-média carotídea.

O procedimento adotado na coleta de dados do EpiFloripa Idoso 2013/2014 incorporou a medida de Espessura Íntima Média das Artérias Carótidas (IMT) das carótidas direitas e esquerdas, com o objetivo de validar o resultado obtido na primeira medida, bem como atestar que os resultados sobre os espessamentos de íntima normalmente se estendem a todas as artérias do sistema circulatório. A equipe responsável por esta coleta realizou procedimentos de treinamento com especialista na área para padronização das medidas a serem realizadas.

As imagens reproduzidas pelo aparelho de USG foram transferidas para um computador que, mediante a leitura dos dados através do software IMT (Intelligence in Medical Technologies), permite a emissão de laudos.

Esta consolidação dos dados foi feita através da digitação dos dados no programa EpiData 3.1, digitadas informações referentes à avaliação dos lados direito e esquerdo das carótidas, compreendendo as variáveis: presença ou não de placas, tamanho das placas $(\mathrm{mm})$, percentual de obstrução, além da extensão medida $(\mathrm{mm})$, qualidade da medida, IMT valor máximo $(\mathrm{mm})$, IMT valor médio $(\mathrm{mm})$ e o desvio padrão $(\mathrm{mm})$. As medidas realizadas da IMT são dadas pelo espaço compreendido entre as duas linhas e corresponde à soma das camadas íntima e média. Adotou-se como critério neste estudo a medida da parede posterior da artéria, uma vez que estudos indicam que as medidas da parede posterior são mais indicativas da real espessura da parede do vaso, além de serem mais reprodutíveis ${ }^{23}$.

\section{Força de preensão manual}

A máxima preensão manual (dinamometria) foi avaliada por meio de dinamômetro mecânico (Takei Kiki Kogyio TK 1201, Japão), ajustado para cada indivíduo, de acordo com o tamanho das mãos.

O teste foi realizado no braço de maior força, de acordo com o relato do participante. Esta informação foi anotada em formulário. Para a execução do teste, o indivíduo permaneceu sentado, com o cotovelo apoiado em uma mesa, antebraço estendido à frente $\mathrm{e}$ palma da mão voltada para cima. O participante foi estimulado a exercer a maior preensão possível. Esse procedimento foi realizado duas vezes, com intervalo de um minuto entre as execuções, e computado o maior valor $(\mathrm{kg})^{24}$.

Para a consolidação do banco de dados, um formulário Google Forms foi criado com campos para preenchimento, com os itens: nome do entrevistado, número de identificação, idade, sexo, membro superior de maior força, mão dominante, mão não dominante e valor da FPM. O formulário foi vinculado a uma planilha no Planilhas Google, para a qual as respostas foram automaticamente enviadas. Ao final das coletas, a planilha foi exportada para o programa Microsoft Office Excel.

Os valores $(\mathrm{kg})$ do desempenho no teste de FPM foram definidos de acordo com o sexo e índice de massa corporal $\left[\mathrm{IMC}=\right.$ massa corporal $(\mathrm{kg}) /$ estatura $\left.(\mathrm{m})^{2}\right]$. Os valores de pontos de corte do IMC foram os seguintes ${ }^{25}$ : IMC $<22 \mathrm{~kg} / \mathrm{m}^{2}$, baixo peso; $\geq 22,0 \mathrm{~kg} / \mathrm{m}^{2}$ e $\leq 27 \mathrm{~kg} / \mathrm{m}^{2}$, peso adequado; e $>27 \mathrm{~kg} / \mathrm{m}^{2}$, excesso de peso. Para cada categoria do IMC, os pontos de corte para a FPM $(\mathrm{kg})$ foram fixados no primeiro quintil, baseado na proposta de Fried et $\mathrm{al}^{26}$ : a) mulheres, $\leq 11,1 \mathrm{~kg}$ para idosas com baixo peso; $\leq 13,5 \mathrm{~kg}$ para idosas com peso adequado; $\mathrm{e} \leq 13,5 \mathrm{~kg}$ para idosas com excesso de peso; b) homens, $\leq 18,0 \mathrm{~kg}$ para idosos com baixo peso; $\leq 21,5 \mathrm{~kg}$ para idosos com peso adequado; e $\leq 23,5 \mathrm{~kg}$ para idosos com excesso de peso.

\section{Atividade física}

As medidas de atividade física e do tempo sedentário foram obtidas por meio do uso de acelerômetro (modelos GT3X e GTX3+) da Actigraf ${ }^{\circledR}$, instrumento válido e fidedigno, que fornece informações quanto ao volume e intensidade das atividades realizadas, expressas em counts $^{27-29}$. Utilizaram-se epochs de 60 segundos, devido ao padrão de atividades de baixa intensidade e longa duração da amostra ${ }^{30,31}$.

Todos os idosos que realizaram os exames foram convidados a utilizar o acelerômetro durante sete dias consecutivos. Porém, indivíduos que apresentassem baixa mobilidade (cadeirantes, acamados, problemas de locomoção), pescadores (por se envolverem em atividades aquáticas) e provável déficit cognitivo não foram considerados elegíveis. Os idosos foram orientados a usar o acelerômetro somente no período de vigília, exceto no banho e em atividades aquáticas. O período de coleta de dados para cada participante teve duração aproximada de 11 dias, correspondendo: a) $1^{\circ}$ dia: programação do monitor e entrega ao pesquisador; b) $2^{\circ}$ dia: entrega do monitor juntamente com um cartão de visita, com número de telefone dos pesquisadores (caso os participantes morassem no mesmo domicílio, os cintos acoplados aos acelerômetros eram distribuídos em cores diferentes) e explicação dos procedimentos de utilização; c) do $3^{\circ}$ ao $9^{\circ}$ dia: período para uso do monitor (programação para coleta com epochs de 60 segundos, iniciada a partir das $00 \mathrm{~h} 00 \mathrm{~min}$ do $3^{\circ} \mathrm{dia}$ ); d) $10^{\circ}$ dia: retirada do monitor; e) $11^{\circ}$ dia: download e validação dos dados.

O controle de qualidade da utilização do equipamento foi realizado por meio de ligações telefônicas no segundo e no quinto dia de uso, com o objetivo de alertar o participante sobre o posicionamento do aparelho (no quadril direito, fixado em um cinto ajustável de tecido de algodão sobre a roupa), sua utilização diária a partir das primeiras horas da manhã, o uso exclusivo, o incentivo à realização das atividades rotineiras, a 
resolução de possíveis dúvidas e sua utilização durante sete dias consecutivos.

A priori, todos os acelerômetros foram calibrados e todos os envolvidos na coleta participaram de treinamento realizado pelos coordenadores da pesquisa, com leitura do manual de instruções. O manual foi disponibilizado a todos os pesquisadores, para que recorressem, a qualquer momento, diante de eventuais dúvidas.

Para diminuir as perdas dos equipamentos pelos participantes e recusas do seu uso, estratégias como oferecimento do transporte até a UFSC ou a busca do aparelho em domicílio, quando observada a impossibilidade de entrega, foram utilizadas. Além disso, valorizou-se a realização de contatos telefônicos educados e gentis em todas as oportunidades, bem como diferentes abordagens que esclarecessem os benefícios da realização dos exames foram mencionadas.

No que se refere à consolidação do banco de dados, os aparelhos foram programados e os dados analisados no software ActiLife versão 6.11.7, e foram consideradas informações válidas quando: a) o participante utilizou o aparelho por pelo menos quatro dias (pelo menos um dia no final de semana); b) apresentou $\geq 600$ minutos de uso/dia em dias de semana e $\geq 480$ minutos/dia no final de semana; e c) horas de uso que registrassem menos de 60 zeros consecutivos $^{32}$. Além disso, adotou-se a seguinte classificação para as intensidades de atividades: a) tempo sedentário ( $\leq 100$ counts $/ \mathrm{min}$ ); b) atividades de intensidade leve (100-1951 counts/min).); c) moderadas ( $\geq 1952$ counts $/ \mathrm{min}$ ); d) vigorosas/muito vigorosas $(\geq 5725 \text { counts } / \mathrm{min})^{29}$.

\section{Suporte técnico geral}

Foram realizadas reuniões semanais entre a coordenadora da pesquisa e os supervisores, cujo objetivo pautava-se na atualização das informações, relato do andamento da coleta e na resolução de dificuldades encontradas, tanto em relação ao manejo dos equipamentos, contato com os idosos, quanto com a exportação e consolidação dos bancos de dados.

\section{Aspectos éticos}

O projeto foi submetido e apreciado conforme a resolução no 466 de 2012 do Conselho Nacional de Saúde, aprovado pelo Comitê de Ética em Pesquisa com Seres Humanos da UFSC, segundo parecer n $n^{\circ} 526.126$, emitido em 09/12/2013. Todos os idosos assinaram o TCLE, que foi entregue em duas vias, após o processo de consentimento em participação da pesquisa.

\section{Financiamento e prestação de contas}

O estudou foi apoiado pelo Conselho Nacional de Desenvolvimento Científico e Tecnológico (CNPq), processo no 475.904/2013-3 e infraestrutura da UFSC. Deste valor, 76,3\% foram aplicados na aquisição de kits e materiais de análises bioquímicas; $5,2 \%$ com pagamento de entrevistadores; $8,5 \%$ despesas administrativas e $10 \%$ em transporte dos voluntários. Por fim, o custo médio por participantes foi de $\mathrm{R} \$ 97,88$ (US\$30,58).

\section{RESULTADOS}

Do total de idosos entrevistados na linha de base, dois foram excluídos por duplicidade de dados, e um devido à idade ser incompatível com o estudo. Assim, foram analisados os resultados de 1.702 idosos, com idade entre 60 e 104 anos (mediana de 70 anos). Para realização do estudo de seguimento, dos 1.702 idosos entrevistados na primeira onda, 217 foram a óbito (12,8\%), 159 foram perdas (9,3\%) e 129 recusas (7,6\%), o que totalizou $1.197(70,3 \%)$ entrevistados. Na etapa dos exames, 10 foram a óbito no período de coleta, 52 foram consideradas perdas, 531 foram consideradas recusas, e aceitaram participar dos exames 604 idosos (taxa de resposta de 50,4\%).

Os idosos que participaram das coletas dos exames eram, na maioria, do sexo feminino, com idade de 60 a 69 anos, brancos, casados(as), com renda superior a 5 salários mínimos, com escolaridade de 1 a 4 anos, com provável ausência de déficit cognitivo, dependência em 1 a 3 atividades da vida diária (AVD) e sem sintomas depressivos (Tabela 1).

Ao comparar as distribuições da linha de base, em relação às características sociodemográficas e às condições de saúde da linha de base e do seguimento com os exames, observou-se diferença entre faixa etária $(\mathrm{p}<0,001)$, estado civil $(\mathrm{p}=0,032)$, estado cognitivo ( $\mathrm{p}<0,001)$, dependência em AVD $(\mathrm{p}=0,009)$ e sintomas depressivos $(\mathrm{p}=0,035)$ (Tabela 1$)$.

Ao ser analisada a distribuição de respondentes, perdas, recusas e óbitos em relação à linha de base, demonstrado na Tabela 2, houve diferenças entre as categorias de faixa etária, raça, estado civil, escolaridade, estado cognitivo, dependência em AVD e sintomas depressivos.

Em relação à faixa etária, os respondentes do seguimento eram idosos mais jovens, e os óbitos ocorreram em 39,1\% daqueles com 80 anos ou mais na linha de base. Na variável raça, houve mais recusas nas categorias de brancos e amarelos. Houve maior percentual de óbitos entre os viúvos, assim como naqueles sem escolaridade formal. Ao analisar a variável déficit cognitivo, percebe-se que houve maior percentual de respondentes entre aqueles que tinham ausência de déficit $\mathrm{e}$ maior percentual de óbitos naqueles com provável déficit. Isso também é observado para a dependência em quatro ou mais AVD, e para com suspeita à depressão, ambos apresentaram maior percentual de óbitos (Tabela 2).

Ao comparar os respondentes com as perdas e recusas agrupadas, sem os óbitos, houve diferença entre as variáveis faixa etária $(\mathrm{p}<0,001)$ e estado cognitivo $(\mathrm{p}<0,001)$. Percebe-se que, com o aumento da idade, houve redução da participação nos exames, e essa redução também foi percebida naqueles com provável déficit cognitivo (Tabela 3 ). 
Tabela 1. Distribuição percentual dos idosos que participaram da coleta, análise e armazenamento de material biológico, exames de imagem e capacidade físico-funcional, segundo características sociodemográficas e de saúde, em relação à primeira onda do Estudo EpiFloripa Idoso $2009 / 2010$

\begin{tabular}{|c|c|c|c|c|c|}
\hline \multirow{2}{*}{ Variáveis } & \multicolumn{2}{|c|}{ Primeira Onda } & \multicolumn{2}{|c|}{ Seguimento } & \multirow{2}{*}{ Valor de } \\
\hline & $\mathbf{n}$ & $\%($ IC95\%) & $\mathbf{n}$ & $\%($ IC95\%) & \\
\hline Sexo & & & & & 0,840 \\
\hline Feminino & 1088 & $62,5(59,6-65,3)$ & 394 & $62,8(58,2-67,3)$ & \\
\hline Masculino & 614 & $37,5(34,7-40,4)$ & 210 & $37,2(32,7-41,8)$ & \\
\hline Total & 1702 & & 604 & & \\
\hline Faixa etária & & & & & $<0,001$ \\
\hline 60 a 69 anos & 851 & $51,0(48,1-53,8)$ & 374 & $62,4(56,9-67,6)$ & \\
\hline 70 a 79 anos & 612 & $35,3(32,5-38,3)$ & 198 & $33,5(28,3-39,2)$ & \\
\hline 80 anos ou mais & 239 & $13,7(11,4-16,4)$ & 32 & $4,1(2,4-6,8)$ & \\
\hline Total & 1702 & & 604 & & \\
\hline Raça & & & & & 0,582 \\
\hline Branca & 1441 & $86,8(83,1-89,7)$ & 505 & $85,7(80,4-89,7)$ & \\
\hline Parta & 131 & $7,3(5,3-9,9)$ & 47 & $7,0(4,5-10,5)$ & \\
\hline Negra ou preta & 84 & $4,3(3,0-6,1)$ & 38 & $5,2(3,2-8,4)$ & \\
\hline Amarela & 12 & $0,6(0,3-1,3)$ & 6 & $0,9(0,3-2,7)$ & \\
\hline Indígena & 17 & $1,0(0,6-1,7)$ & 5 & $1,2(0,5-3,1)$ & \\
\hline Total & 1685 & & 601 & & \\
\hline Estado civil & & & & & 0,032 \\
\hline Casado(a) & 990 & $58,3(54,7-61,8)$ & 364 & $62,3(56,9-67,5)$ & \\
\hline Solteiro(a) & 99 & $5,4(4,1-7,2)$ & 40 & $5,9(3,7-9,3)$ & \\
\hline Divorciado/Separado/Desquitado(a) & 132 & $8,3(6,9-10,0)$ & 53 & $9,5(6,8-13,2)$ & \\
\hline Viúvo(a) & 481 & $27,9(25,0-31,0)$ & 147 & $22,2(18,5-26,5)$ & \\
\hline Total & 1702 & & 604 & & \\
\hline Renda familiar & & & & & 0,468 \\
\hline Menor ou igual a $1 \mathrm{SM}$ & 238 & $12,6(10,3-15,5)$ & 77 & $11,7(8,5-16,0)$ & \\
\hline$>1$ a $3 \mathrm{SM}$ & 429 & $25,2(21,4-29,4)$ & 141 & $22,9(17,8-28,9)$ & \\
\hline$>3 \mathrm{SM}$ a $5 \mathrm{SM}$ & 309 & $17,7(15,6-20,0)$ & 119 & $19,7(15,5-24,6)$ & \\
\hline$>5 \mathrm{SM}$ a $10 \mathrm{SM}$ & 393 & $23,7(20,8-26,8)$ & 148 & $25,2(21,4-29,4)$ & \\
\hline$>10 \mathrm{SM}$ & 333 & $20,8(17,0-25,1)$ & 119 & $20,5(16,0-25,8)$ & \\
\hline Total & 1702 & & 604 & & \\
\hline Escolaridade & & & & & 0,088 \\
\hline Sem escolaridade formal & 158 & $8,2(6,3-10,5)$ & 42 & $5,8(4,1-8,1)$ & \\
\hline 1 a 4 anos & 595 & $33,1(28,6-37,9)$ & 218 & $33,9(26,5-42,1)$ & \\
\hline 5 a 8 anos & 307 & $17,8(15,4-20,4)$ & 107 & $17,9(14,3-22,0)$ & \\
\hline 9 a 11 anos & 241 & $16,0(13,6-18,8)$ & 94 & $18,9(15,2-22,7)$ & \\
\hline 12 ou mais anos & 393 & $25,0(20,4-30,2)$ & 142 & $23,8(18,8-29,6)$ & \\
\hline Total & 1694 & & 603 & & \\
\hline Estado cognitivo $^{*}$ & & & & & $<0,001$ \\
\hline Ausência de provável déficit cognitivo & 1244 & $76,0(71,2-80,2)$ & 495 & $84,1(78,3-88,6)$ & \\
\hline Presença de provável déficit cognitivo & 443 & $24,0(20,0-28,8)$ & 106 & $15,9(11,4-21,7)$ & \\
\hline Total & 1687 & & 601 & & \\
\hline \multicolumn{3}{|c|}{ Dependência em Atividades de Vida Diária (AVD) $\dagger$} & & & 0,009 \\
\hline Nenhuma AVD & 457 & $25,6(22,3-29,1)$ & 194 & $30,4(25,0-36,4)$ & \\
\hline 1 a 3 AVD & 708 & $43,0(38,9-47,2)$ & 260 & $43,9(38,4-49,5)$ & \\
\hline 4 ou mais AVD & 537 & $31,4(28,3-34,8)$ & 150 & $25,7(21,7-30,2)$ & \\
\hline Total & 1702 & & 604 & & \\
\hline Sintomas depressivos & & & & & 0,035 \\
\hline Normal & 1409 & $86,4(84,0-88,4)$ & 523 & $89,8(85,3-93,1)$ & \\
\hline Suspeita à depressão & 225 & $13,6(11,5-16,10)$ & 63 & $10,2(6,9-14,7)$ & \\
\hline Total & 1634 & & 586 & & \\
\hline
\end{tabular}

* Investigado pelo Miniexame do Estado Mental ${ }^{33}$, categorizado de acordo com Almeida ${ }^{34}$; $\dagger$ Avaliada por meio do Questionário Brasileiro de Avaliação Funcional Multidimensional adaptado do questionário Old Americans Resources and Services (BOMFAQ/OARS), categorizada de acordo com Rosa et al. ${ }^{35}$ 


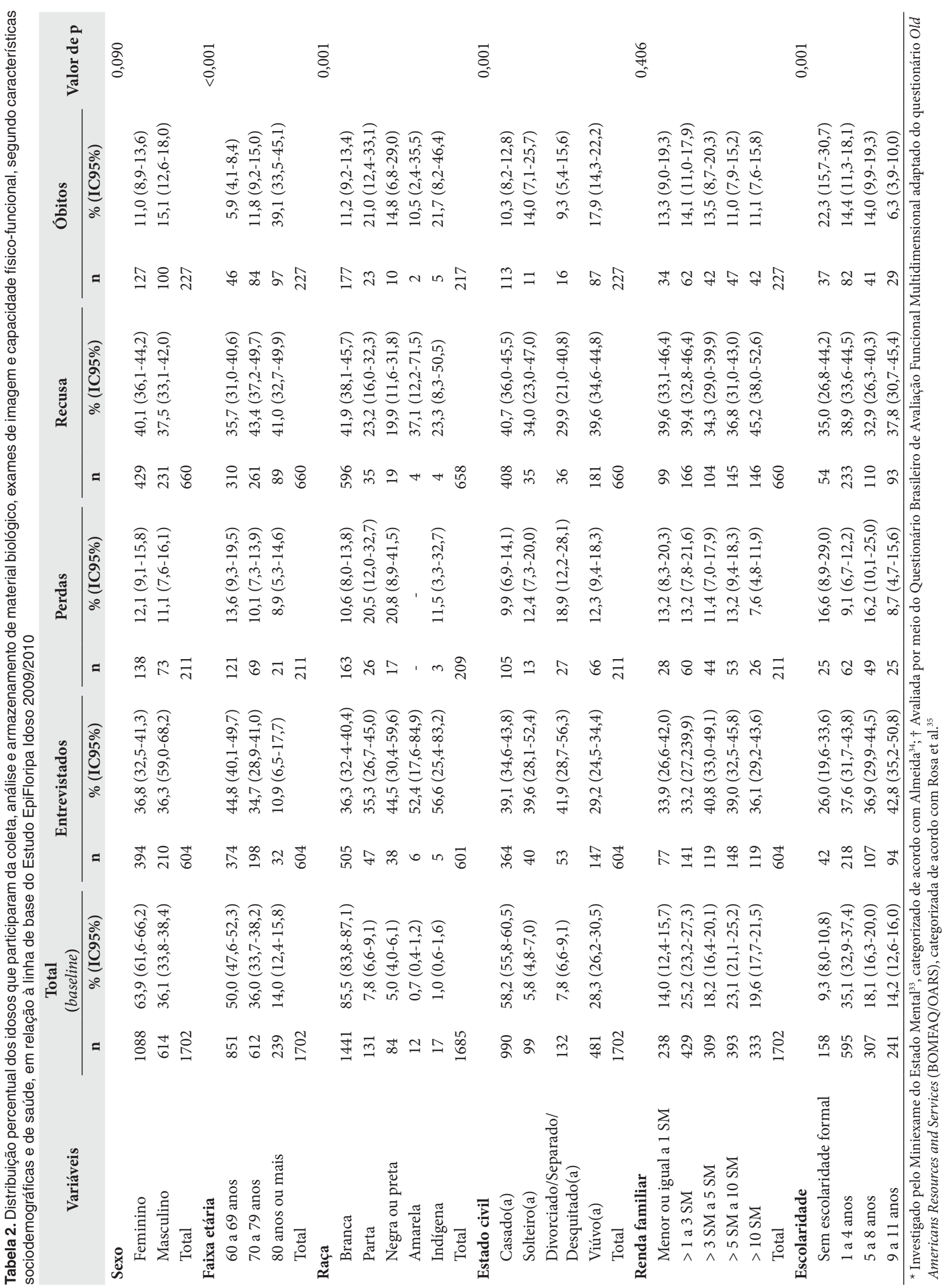




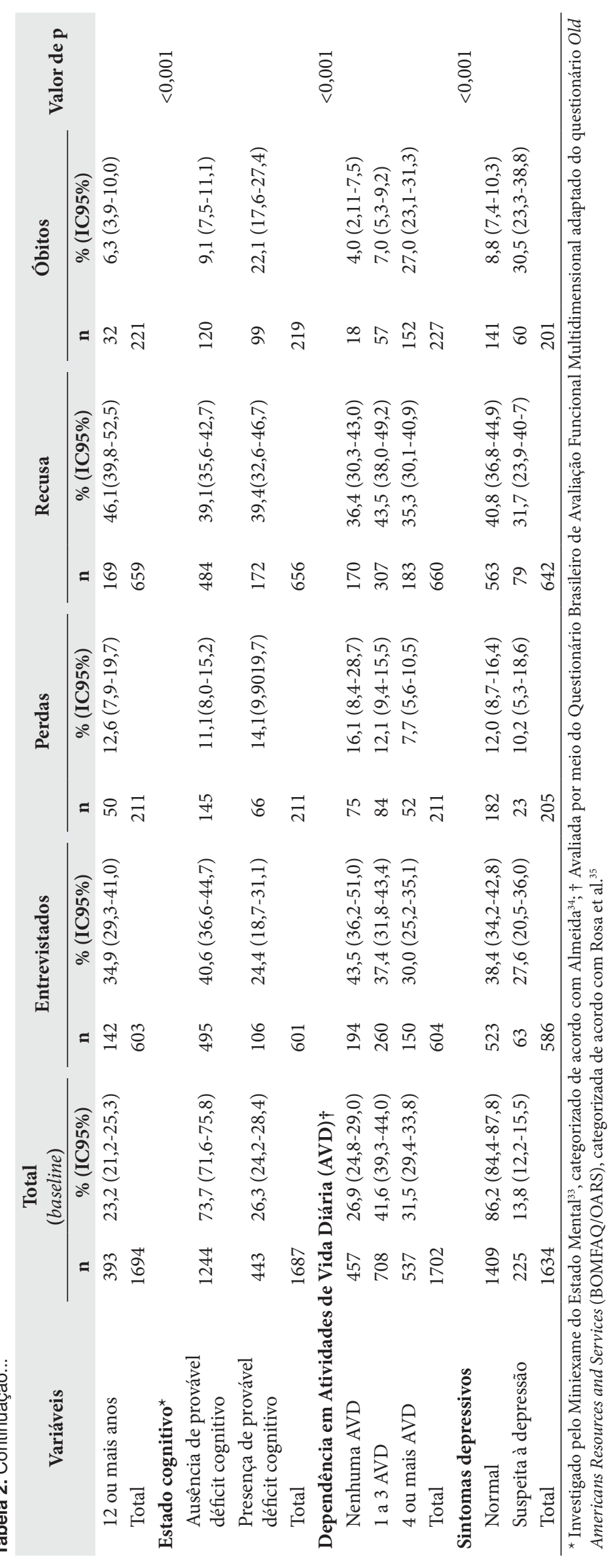


Tabela 3. Distribuição percentual dos idosos que participaram da coleta, análise e armazenamento de material biológico, exames de imagem e capacidade físico-funcional, segundo características solciodemográficas e de saúde, em relação à linha de baseado Estudo EpiFloripa Idoso 2009/2010

\begin{tabular}{|c|c|c|c|c|c|}
\hline \multirow{2}{*}{ Variáveis } & \multicolumn{2}{|c|}{ Agendado/fizeram exame } & \multicolumn{2}{|c|}{ Não resposta (perdas/recusa) } & \multirow{2}{*}{ Valor de $\mathrm{p}$} \\
\hline & $\mathbf{n}$ & $\%(\mathrm{IC} 95 \%)$ & $\mathbf{n}$ & $\%($ IC95\%) & \\
\hline Sexo & & & & & 0,644 \\
\hline Feminino & 394 & $41,4(36,7-46,2)$ & 567 & $58,6(53,8-63,3)$ & \\
\hline Masculino & 210 & $42,7(37,3-48,3)$ & 304 & $57,3(51,7-62,6)$ & \\
\hline Total & 604 & & 871 & & \\
\hline Faixa etária & & & & & $<0,001$ \\
\hline 60 a 69 anos & 374 & $47,6(42,4-52,9)$ & 431 & $52,4(47,1-57,6)$ & \\
\hline 70 a 79 anos & 198 & $39,4(32,7-46,4)$ & 330 & $60,6(53,6-67,3)$ & \\
\hline 80 anos ou mais & 32 & $17,9(10,6-28,8)$ & 110 & $82,1(71,2-89,4)$ & \\
\hline Total & 604 & & 871 & & \\
\hline Raça & & & & & 0,418 \\
\hline Branca & 505 & $40,9(36,5-45,5)$ & 759 & $59,1(54,5-63,5)$ & \\
\hline Parta & 47 & $44,7(34,5-55,4)$ & 61 & $55,3(44,6-65,5)$ & \\
\hline Negra ou preta & 38 & $52,3(35,8-68,2)$ & 36 & $47,7(31,8-64,2)$ & \\
\hline Amarela & 6 & $58,5(23,4-86,7)$ & 4 & $41,5(13,3-76,6)$ & \\
\hline Indígena & 5 & $55,5(24,5-82,7)$ & 7 & $44,5(17,3-75,5)$ & \\
\hline Total & 601 & & 867 & & \\
\hline Estado civil & & & & & 0,178 \\
\hline Casado(a) & 364 & $43,6(38,4-49,0)$ & 513 & $56,4(51,0-61,6)$ & \\
\hline Solteiro(a) & 40 & $46,1(33,8-58,8)$ & 48 & $53,9(41,1-66,2)$ & \\
\hline Divorciado/Separado/Desquitado(a) & 53 & $46,2(32,2-60,8)$ & 63 & $53,8(39,2-67,8)$ & \\
\hline Viúvo(a) & 147 & $35,5(30,2-41,2)$ & 247 & $64,5(58,8-69,8)$ & \\
\hline Total & 604 & & & & \\
\hline Renda familiar & & & & & 0,455 \\
\hline Menor ou igual a $1 \mathrm{SM}$ & 77 & $39,1(31,73-47,0)$ & 127 & $60,9(53,0-68,3)$ & \\
\hline$>1$ a $3 \mathrm{SM}$ & 141 & $38,7(31,27-46,7)$ & 226 & $61,3(53,3-68,7)$ & \\
\hline$>3 \mathrm{SM}$ a $5 \mathrm{SM}$ & 119 & $47,2(39,17-55,3)$ & 148 & $52,8(44,7-60,8)$ & \\
\hline$>5 \mathrm{SM}$ a $10 \mathrm{SM}$ & 148 & $43,8(36,98-50,8)$ & 198 & $56,2(49,2-63,0)$ & \\
\hline$>10 \mathrm{SM}$ & 119 & $40,6(32,60-49,1)$ & 172 & $59,4(50,9-67,4)$ & \\
\hline Total & 604 & & 871 & & \\
\hline Escolaridade & & & & & 0,130 \\
\hline Sem escolaridade formal & 42 & $33,5(25,5-42,6)$ & 79 & $66,5(57,4-74,5)$ & \\
\hline 1 a 4 anos & 218 & $43,9(37,3-50,7)$ & 295 & $56,1(49,3-62,7)$ & \\
\hline 5 a 8 anos & 107 & $42,9(34,4-51,8)$ & 159 & $57,1(48,1-65,6)$ & \\
\hline 9 a 11 anos & 94 & $48,0(39,6-56,4)$ & 118 & $52,0(43,6-60,4)$ & \\
\hline 12 ou mais anos & 142 & $37,3(31,1-43,9)$ & 219 & $62,7(56,1-68,9)$ & \\
\hline Total & 603 & & & & \\
\hline Estado cognitivo $^{\star}$ & & & & & 0,001 \\
\hline Ausência de provável déficit cognitivo & 495 & $44,7(40,4-49,0)$ & 629 & $55,3(51,0-59,6)$ & \\
\hline Presença de provável déficit cognitivo & 106 & $31,3(24,2-39,4)$ & 238 & $68,7(60,6-75,8)$ & \\
\hline Total & 601 & & & & \\
\hline Dependência em Atividades de Vida Di & D) $\dagger$ & & & & 0,502 \\
\hline Nenhuma AVD & 194 & $45,3(37,5-53,3)$ & 245 & $54,7(46,7-62,4)$ & \\
\hline 1 a 3 AVD & 260 & $40,2(34,3-46,4)$ & 391 & $59,8(53,6-65,6)$ & \\
\hline 4 ou mais AVD & 150 & $41,0(34,6-47,8)$ & 235 & $59,0(52,2-65,4)$ & \\
\hline Total & 604 & & 871 & & \\
\hline Sintomas depressivos & & & & & 0,705 \\
\hline Normal & 523 & $42,1(37,6-46,8)$ & 745 & $57,9(53,2-62,4)$ & \\
\hline Suspeita à depressão & 63 & $39,7(28,9-51,6)$ & 102 & $60,9(48,4-71,1)$ & \\
\hline Total & 586 & & 847 & & \\
\hline
\end{tabular}

* Investigado pelo Miniexame do Estado Mental ${ }^{33}$, categorizado de acordo com Almeida ${ }^{34}$; $\dagger$ Avaliada por meio do Questionário Brasileiro de Avaliação Funcional Multidimensional adaptado do questionário Old Americans Resources and Services (BOMFAQ/OARS), categorizada de acordo com Rosa et al. ${ }^{35}$ 


\section{DISCUSSÃO}

Com base nos resultados encontrados, foi possível observar que os idosos que realizaram os exames, quando comparados à distribuição da linha de base, apresentaram mudanças importantes na faixa etária, estado civil, estado cognitivo, dependência funcional e sintomas depressivos. Ainda quando analisada a distribuição entre os respondentes, perdas, recusas e óbitos, os idosos mais velhos, viúvos, sem escolaridade formal, com provável déficit cognitivo, com dependência em quatro ou mais AVD e com suspeita de depressão foram mais a óbito. Além disso, observou-se que os idosos mais velhos e com déficit cognitivo reduziram a participação nos exames.

$\mathrm{O}$ fato de idosos mais velhos terem maior prevalência de mortalidade quando comparados a idosos mais novos é esperado ${ }^{36}$, visto o curso natural da vida, maior probabilidade de doenças, menor nível de atividade física, redução da capacidade física e mental e, consequentemente, pior qualidade de vida ${ }^{37}$.

$\mathrm{O}$ estado civil tem papel significativo nos diferenciais de saúde, pois foi demonstrado que o casamento tem efeito protetivo para bons hábitos de saúde ${ }^{36}$. A viuvez tem relação com a maior mortalidade, dada à influência negativa sobre o estado de saúde, luto, sofrimento psíquico, transtornos mentais e menor utilização de serviços de saúde ${ }^{38}$.

Quanto à escolaridade, constatou-se que idosos com menores níveis educacionais morreram mais. Piores condições de moradia, de acesso à saúde, acesso a informações, menor renda e maior risco de quedas podem estar relacionados à escolaridade ${ }^{39,40}$.

Os resultados também evidenciaram que os idosos dependentes em quatro ou mais AVD, assim como aqueles com provável déficit cognitivo, tiveram maiores proporções de óbitos quando comparados aos independentes e àqueles sem alterações cognitivas, respectivamente. De acordo com os dados do National Health and Nutrition Examination Survey (NHANES), a presença de incapacidade no domínio de atividades básicas da vida diária, por si só, mostrou chances quase três vezes maiores de mortalidade por todas as causas de morte entre os idosos americanos com idade entre 65 e 84 anos, independente das suas características sociodemográficas e demais condições de saúde ${ }^{41}$. De modo semelhante, o pior desempenho em testes cognitivos, quando avaliado isoladamente, correlaciona-se inversamente ao maior risco de mortalidade em idosos a partir de 60 anos $^{42}$.

Além desses, outros estudos longitudinais têm evidenciado que o risco de mortalidade se eleva substancialmente na presença de incapacidades funcionais e cognitivas ${ }^{43,44}$. Explicações plausíveis para essas associações são multifatoriais e envolvem, especialmente, a perda de força e/ou massa muscular e a menor velocidade de marcha, as quais decorrem tanto das alterações funcionais e cognitivas quanto do próprio avanço da idade ${ }^{45}$. Com isso, torna-se claro que a deteç̧ão precoce, tanto da incapacidade quanto do déficit cognitivo, pode ser benéfica para reconhecer quais idosos mais necessitam de intervenções que visem diminuir o risco de morte.
A maior mortalidade de idosos com suspeita de depressão tem sido atribuída ao fato de que idosos com sintomas depressivos têm pior qualidade de vida e aumento do risco para doenças cardiovasculares e demência ${ }^{46,47}$. Em Taiwan, após oito anos de acompanhamento de uma coorte de 2.416 idosos, foi possível detectar maior risco para mortalidade entre aqueles com sintomas depressivos, em especial do sexo masculino. Do mesmo modo, estudo com idosos na Dinamarca também identificou associação entre a presença de sintomas depressivos e maior taxa de mortalidade entre indivíduos com demência ${ }^{48}$. No Brasil, estudo com dados do Sistema de Informação sobre Mortalidade do estado da Bahia também identificou relação entre a presença de sintomas depressivos e mortalidade em idosos ${ }^{49}$. O incentivo para a adoção de medidas que estimulem a qualidade de vida pode contribuir substancialmente para a redução na ocorrência de sintomas depressivos e, por conseguinte, da mortalidade entre idosos.

Os achados que os idosos mais velhos e com provável déficit cognitivo participaram menos da etapa dos exames estão em consonância com o estudo de coorte realizado na região Sudeste do Brasil ${ }^{50}$. O aumento da idade mostra-se fator associado ao déficit cognitivo ${ }^{8}$, e essas variáveis também estão associadas à redução da capacidade funcional ${ }^{51}$, perda de independência ${ }^{52}$ e autonomia do idoso, o que pode explicar a menor participação em pesquisas que exijam deslocamento do avaliado.

Considera-se ainda que a idade é uma variável importante, não por ser causal, mas por indicar um conjunto de outras influências relacionadas a alterações comportamentais ${ }^{53}$, as quais podem ter influenciado a não participação dos idosos mais velhos. Além disso, pode-se considerar também o viés de sobrevivência, no qual pessoas com pior situação socioeconômica e com maiores exposições de risco tendem a morrer mais cedo ou não participar de estudos, visto as dificuldades de sair de casa $^{54}$. Uma das estratégias adotadas a fim de reduzir o viés foi a disponibilização do transporte, adaptado, para os participantes e acompanhantes.

O desenvolvimento desse estudo exigiu planejamento, estrutura física, recursos financeiros, além de equipe multidisciplinar. Apesar da complexidade da execução, alguns aspectos merecem ser mencionados como facilitadores, tais como: envolvimento da equipe, projetos de pesquisa de interesse individuais atrelados às variáveis coletadas; a troca de experiências e resolução de problemas oportunizada por diferentes formações, e a divisão de tarefas entre todos os pesquisadores, o que melhorou a logística e evitou a sobrecarga de trabalho.

Como aspecto negativo, ressalta-se a indisponibilidade de um dos laboratórios para todos os dias úteis, além de laboratórios com maior infraestrutura que possibilitassem a execução de mais procedimentos e maior volume de pessoas atendidas, o que reduziria o tempo de coleta de dados. 
Cabe destacar também que a taxa de resposta do estudo foi baixa $(50,4 \%)$, e isso pode ter ocorrido por diversos fatores, dentre eles a dificuldade dos idosos de saírem de suas residências para participarem da pesquisa. Nesse sentido, o cenário ideal, para abranger um grande número de idosos participantes, seria necessário que esses exames fossem feitos nos domicílios dos idosos, o que é inviável pelos equipamentos utilizados na pesquisa e em razão baixo financiamento para a realização do estudo.
Espera-se que a descrição das rotinas utilizadas nesta pesquisa possa auxiliar no desenvolvimento de novas pesquisas baseadas em coleta, análise e armazenamento de material biológico, exames de imagem e da capacidade físico-funcional em outros grupos de idosos. Estes tipos de investigações utilizam métodos de avaliação direta, que podem diagnosticar fatores de risco e proteção de diversas doenças, com muita eficiência, validade e confiabilidade, auxiliando assim na saúde das pessoas idosas.

\section{REFERÊNCIAS}

1. Malta DC, Leal MC, Costa MFL, Morais OL No. Inquéritos Nacionais de Saúde: experiência acumulada e proposta para o inquérito de saúde brasileiro. Rev Bras Epidemiol. 2008;11(Suppl 1):159-67. http://dx.doi. org/10.1590/S1415-790X2008000500017.

2. Fedeli LG, Vidigal PG, Leite CM, Castilhos CD, Pimentel RA, Maniero $\mathrm{VC}$, et al. Logística de coleta e transporte de material biológico e organização do laboratório central no ELSA-Brasil. Rev Saude Publica. 2013;47(Suppl 2):6371. http://dx.doi.org/10.1590/S0034-8910.2013047003807. PMid:24346722.

3. Mahmood SS, Levy D, Vasan RS, Wang TJ. The Framingham Heart Study and the epidemiology of cardiovascular disease: a historical perspective. Lancet. 2014;383(9921):999-1008. http://dx.doi.org/10.1016/S01406736(13)61752-3. PMid:24084292.

4. Banks J, Batty GD, Begum N, Demakakos P, Oliveira C, Head J, et al. The dynamics of ageing: Evidence from the English Longitudinal Study of Ageing 2002-15 (Wave 7). Londres: The Institute for Fiscal Studies; 2016.

5. Brasil. Ministério da Saúde. Secretaria de Vigilância em Saúde. VIGITEL Brasil 2008: vigilância de fatores de risco e proteção para doenças crônicas por inquérito telefônico. Brasília: Ministério da Saúde; 2009.

6. Rothman KJ. Epidemiology - An Introduction. 2nd ed. New York: Oxford; 2012 .

7. Mill JG, Pinto K, Griep RH, Goulart A, Foppa M, Lotufo PA, et al. Aferições e exames clínicos realizados nos participantes do ELSA-Brasil. Rev Saude Publica. 2013;47(Suppl 2):54-62. http://dx.doi.org/10.1590/ S0034-8910.2013047003851. PMid:24346721.

8. Lebrão ML, Laurenti R. Saúde, bem-estar e envelhecimento: o estudo SABE no Município de São Paulo. Rev Bras Epidemiol. 2005;8(2):127-41. http:// dx.doi.org/10.1590/S1415-790X2005000200005.

9. Lima-Costa M. Estudo de coorte de idosos de Bambuí (1997-2008). Cad Saude Publica. 2011;27(Suppl 3):S324-5. http://dx.doi.org/10.1590/S0102311X2011001500001. PMid:21952852.

10. Ramos LR. Fatores determinantes do envelhecimento saudável em idosos residentes em centro urbano: Projeto Epidoso, São Paulo Determinant factors for healthy aging among senior citizens in a large city: the Epidoso. Cad Saude Publica. 2003;19(3):793-8. http://dx.doi.org/10.1590/S0102311X2003000300011. PMid:12806481.

11. Atlas do Desenvolvimento Humano no Brasil. Programa das Nações Unidas. Instituto de Pesquisa Econômica Aplicada. Fundação João Pinheiro [Internet]. Brasília: PNUD:IPEA:FJP; 2018 [cited 2018 Aug 29]. Available from: http://www.atlasbrasil.org.br/2013/pt

12. Atlas do Desenvolvimento Humano no Brasil. Programa das Nações Unidas. Instituto de Pesquisa Econômica Aplicada. Fundação João Pinheiro
[Internet]. 2013 [cited 2018 Aug 29]. Available from: http://www.atlasbrasil. org.br/2013/pt/perfil_m/florianopolis_sc.

13. Brasil. Ministério da Saúde. Informações de Saúde (TABNET). Índice de GINI da renda domiciliar per capita [Internet]. Brasília: IBGE; 2010 [cited 2018 Aug 29]. Available from: http://tabnet.datasus.gov.br/cgi/ibge/censo/ cnv/ginibr.def

14. Confortin SC, Schneider IJC, Antes DL, Cembranel F, Ono LM, Marques LP, et al. Life and health conditions among elderly: results of the EpiFloripa Idoso cohort study. Epidemiol Serv Saude. 2017;26(2):305-17. http://dx.doi. org/10.5123/S1679-49742017000200008. PMid:28492772.

15. Xavier H, Izar M, Faria J No, Assad M, Rocha V, Sposito A, et al. V Diretriz brasileira de dislipidemias e prevenção da aterosclerose. Arq Bras Cardiol. 2013;101(4):1-20. http://dx.doi.org/10.5935/abc.2013S010. PMid:24217493.

16. Castelli WP. Cholesterol and lipids in the risk of coronary artery disease: The Framingham Heart Study. Can J Cardiol. 1988;4(Suppl A):5A-10A. PMid:3179802.

17. Zinman B, Gerich J, Buse JB, Lewin A, Schwartz S, Raskin P, et al. American Diabetes Association. Standards of medical care in diabetes-2010. Diabetes Care. 2010;33(Suppl 1):S11-61. http://dx.doi.org/10.2337/dc10-S011.

18. National Glycohemoglobin Standardization Program. IFCC standardization of HbA1C [Internet]. 2018 [cited 2018 Aug 24]. Available from: http:// www.ngsp.org/docs/IFCCstd.pdf

19. Wintrobe MM, Lee GR. Wintrobe's clinical haematology. 10th ed. Oxford: Blackwell Science Ltd; 1999. 2763 p.

20. Pearson TA, Mensah GA, Alexander RW, Anderson JL, Cannon RO 3rd, Criqui $\mathrm{M}$, et al. Markers of inflammation and cardiovascular disease application to clinical and public health practice: a statement for healthcare professionals from the centers for disease control and prevention and the American Heart Association. Circulation. 2003;107(3):499-511. http:// dx.doi.org/10.1161/01.CIR.0000052939.59093.45. PMid:12551878.

21. Genton L, Hans D, Kyle UG, Pichard C. Dual-energy X-ray absorptiometry and body composition: differences between devices and comparison with reference methods. Nutrition. 2002;18(1):66-70. http://dx.doi.org/10.1016/ S0899-9007(01)00700-6. PMid:11827768.

22. Kanis JA, Melton LJ 3rd, Christiansen C, Johnston CC, Khaltaev N. The diagnosis of osteoporosis. J Bone Miner Res. 1994;9(8):1137-41. http:// dx.doi.org/10.1002/jbmr.5650090802. PMid:7976495.

23. Stensland-Bugge E, Bønaa KH, Joakimsen O. Reproducibility of ultrasonographically determined intima-media thickness is dependent on arterial wall thickness the tromsø study. Stroke. 1997;28(10):1972-80. http://dx.doi.org/10.1161/01.STR.28.10.1972. PMid:9341706. 
24. Barbosa AR, Souza JM, Lebrão ML, Laurenti R, Marucci MFN. Functional limitations of Brazilian elderly by age and gender differences: data from SABE Survey. Cad Saude Publica. 2005;21(4):1177-85. http://dx.doi. org/10.1590/S0102-311X2005000400020. PMid:16021255.

25. American Academy of Family Physicians, American Dietetic Association, National Council on the Aging. Nutrition screening intervention resources for healthcare professionals working with older adults. Nutrition Screening Initiative. Washington: American Dietetic Association; 2002 [cited 2018 Aug 29]. Available from: http://www.eatright.org.

26. Fried LP, Tangen CM, Walston J, Newman AB, Hirsch C, Gottdiener J, et al. Frailty in older adults evidence for a phenotype. J Gerontol A Biol Sci Med Sci. 2001;56(3):M146-57. http://dx.doi.org/10.1093/gerona/56.3.M146. PMid:11253156.

27. Keadle SK, Shiroma EJ, Freedson PS, Lee I-M. Impact of accelerometer data processing decisions on the sample size, wear time and physical activity level of a large cohort study. BMC Public Health. 2014;14(1):1. http:// dx.doi.org/10.1186/1471-2458-14-1210. PMid:25421941.

28. Evenson KR, Buchner DM, Morland KB. Objective measurement of physical activity and sedentary behavior among US adults aged 60 years or older. Prev Chronic Dis. 2012;9:E26. PMid:22172193.

29. Sasaki JE, John D, Freedson PS. Validation and comparison of ActiGraph activity monitors. J Sci Med Sport. 2011;14(5):411-6. http://dx.doi. org/10.1016/j.jsams.2011.04.003. PMid:21616714.

30. Freedson PS, Melanson E, Sirard J. Calibration of the computer science and applications, Inc. accelerometer. Med Sci Sports Exerc. 1998;30(5):777-81. http://dx.doi.org/10.1097/00005768-199805000-00021. PMid:9588623.

31. Gorman E, Hanson H, Yang P, Khan K, Liu-Ambrose T, Ashe M. Accelerometry analysis of physical activity and sedentary behavior in older adults: a systematic review and data analysis. Eur Rev Aging Phys Act. 2014;11(1):35-49. http://dx.doi.org/10.1007/s11556-013-0132-x. PMid:24765212.

32. Sallis JF, Saelens BE, Frank LD, Conway TL, Slymen DJ, Cain KL, et al. Neighborhood built environment and income: Examining multiple health outcomes. Soc Sci Med. 2009;68(7):1285-93. http://dx.doi.org/10.1016/j. socscimed.2009.01.017. PMid:19232809.

33. Folstein MF, Folstein SE, McHugh PR. "Mini-mental state". A practical method for grading the cognitive state of patients for the clinician. J Psychiatr Res. 1975;12(3):189-98. http://dx.doi.org/10.1016/0022-3956(75)90026-6. PMid:1202204.

34. Almeida OP. Mini exame do estado mental e o diagnóstico de demência no Brasil. Arq Neuropsiquiatr. 1998;56(3B):605-12. http://dx.doi.org/10.1590/ S0004-282X1998000400014. PMid:9850757.

35. Rosa TE, Benício MH, Latorre MR, Ramos LR. Fatores determinantes da capacidade funcional entre idosos. Rev Saude Publica. 2003;37(1):40-8. http://dx.doi.org/10.1590/S0034-89102003000100008. PMid:12488919.

36. Oliveira TC, Medeiros WR, Lima KC. Diferenciais sócio-demográficos da mortalidade de idosos em idades precoces e longevas. Rev Baiana Saúde Pública. 2015;59(2):249-61.

37. Mechling H, Netz Y. Aging and inactivity-capitalizing on the protective effect of planned physical activity in old age. Eur Rev Aging Phys Act. 2009;6(2):89-97. http://dx.doi.org/10.1007/s11556-009-0052-y.

38. Fernandes BL, Borgato MH. A Viuvez e a Saúde dos Idosos: uma Revisão Integrativa. Kairós Gerontol. 2016;19(3):18.
39. Pereira GN, Morsch P, Lopes DGC, Trevisan MD, Ribeiro A, Navarro $\mathrm{JHN}$, et al. Fatores socioambientais associados à ocorrência de quedas em idosos. Cien Saude Colet. 2013;18(12):3507-14. http://dx.doi.org/10.1590/ S1413-81232013001200007. PMid:24263867.

40. Jaffe DH, Neumark YD, Eisenbach Z, Manor O. Parity-related mortality: shape of association among middle-aged and elderly men and women. Eur J Epidemiol. 2009;24(1):9-16. http://dx.doi.org/10.1007/s10654-008-9310-y. PMid:19145406.

41. Wu LW, Chen WL, Peng TC, Chiang ST, Yang HF, Sun YS, et al. All-cause mortality risk in elderly individuals with disabilities: a retrospective observational study. BMJ Open. 2016;6(9):e011164. http://dx.doi.org/10.1136/ bmjopen-2016-011164. PMid:27625055.

42. Obisesan TO, Gillum RF. Cognitive function, social integration and mortality in a U.S. national cohort study of older adults. BMC Geriatr. 2009;9(1):33. http://dx.doi.org/10.1186/1471-2318-9-33. PMid:19638207.

43. Millán-Calenti JC, Tubio J, Pita-Fernandez S, Gonzalez-Abraldes I, Lorenzo T, Fernandez-Arruty T, et al. Prevalence of functional disability in Activities of Daily Living (ADL), Instrumental Activities of Daily Living (IADL) and associated factors, as predictors of morbidity and mortality. Arch Gerontol Geriatr. 2010;50(3):306-10. http://dx.doi.org/10.1016/j.archger.2009.04.017. PMid:19520442.

44. Landi F, Liperoti R, Russo A, Capoluongo E, Barillaro C, Pahor M, et al. Disability, more than multimorbidity, was predictive of mortality among older persons aged 80 years and older. J Clin Epidemiol. 2010;63(7):752-9. http://dx.doi.org/10.1016/j.jclinepi.2009.09.007. PMid:20056387.

45. Legrand D, Vaes B, Mathei C, Adriaensen W, Van Pottelbergh G, Degryse JM. Muscle strength and physical performance as predictors of mortality, hospitalization, and disability in the oldest old. J Am Geriatr Soc. 2014;62(6):1030-8. http://dx.doi.org/10.1111/jgs.12840. PMid:24802886.

46. Peters R, Pinto E, Beckett N, Swift C, Potter J, McCormack T, et al Association of depression with subsequent mortality, cardiovascular morbidity and incident dementia in people aged 80 and over and suffering from hypertension. Data from the Hypertension in the Very Elderly Trial (HYVET). Age Ageing. 2010;39(4):439-45. http://dx.doi.org/10.1093/ ageing/afq042. PMid:20497949.

47. Teng PR, Yeh CJ, Lee MC, Lin HS, Lai TJ. Depressive symptoms as an independent risk factor for mortality in elderly persons: results of a national longitudinal study. Aging Ment Health. 2013;17(4):470-8. http://dx.doi.or g/10.1080/13607863.2012.747081. PMid:23215855.

48. Petersen JD, Waldorff FB, Siersma VD, Phung TKT, Bebe ACKM, Waldemar G. Major depressive symptoms increase 3-Year Mortality Rate in patients with mild dementia. Int J Alzheimers Dis. 2017;2017:8. http://dx.doi. org/10.1155/2017/7482094. PMid:28484660.

49. Santos PHS, Carmo ÉA, Ribeiro BS, Soares CJ, Santana MLAD, et al Perfil da mortalidade por depressão em idosos no estado da Bahia. Kairós Gerontol. 2016;19(3):12.

50. Ramos LR, Toniolo NJ, Cendoroglo MS, Garcia JT, Najas MS, Perracini M, et al. Two-year follow-up study of elderly residents in São Paulo, Brazil: methodology and preliminary results. Rev Saude Publica. 1998;32(5):397407. http://dx.doi.org/10.1590/S0034-89101998000500001. PMid:10030055.

51. Oliveira SFD, Duarte YAO, Lebrão ML, Laurenti R. Demanda referida e auxílio recebido por idosos com declínio cognitivo no município de 
São Paulo. Saude Soc. 2007;16(1):81-9. http://dx.doi.org/10.1590/S010412902007000100008 .

52. Tonelli M, Wiebe N, Straus S, Fortin M, Guthrie B, James MT, et al. Multimorbidity, dementia and health care in older people:a population-based cohort study. CMAJ Open. 2017;5(3):E623-e31. http://dx.doi.org/10.9778/ cmajo.20170052. PMid:28811281.

53. Batistoni SST, Neri AL, Cupertino APFB. Medidas prospectivas de sintomas depressivos entre idosos residentes na comunidade. Rev Saude Publica. 2010;44(6):1137-43. http://dx.doi.org/10.1590/S0034-89102010000600020. PMid:21107509.
54. Berkman CS, Gurland BJ. The relationship among income, other socioeconomic indicators, and functional level in older persons. J Aging Health. 1998;10(1):81-98. http://dx.doi.org/10.1177/089826439801000105. PMid:10182419.

55. American Diabetes Association. Standards of medical care in diabetes-2010. Diabetes Care. 2010;33(Suppl 1):S11-61. http://dx.doi.org/10.2337/dc10-S011. PMid:20042772.

Recebido em: Maio 29, 2017 Aceito em: Dez. 18, 2018 